\title{
Poor outcome of hepatocellular carcinoma with stemness marker under hypoxia: resistance to transarterial chemoembolization
}

\author{
Hyungjin Rhee ${ }^{1}$, Ji Hae Nahm ${ }^{1}$, Haeryoung $\mathrm{Kim}^{2}$, Gi Hong $\mathrm{Choi}^{3}$, Jeong Eun Yoo ${ }^{1}$, \\ Hye Sun Lee ${ }^{4}$, Myoung Ju Koh ${ }^{1}$ and Young Nyun Park ${ }^{1,5}$ \\ ${ }^{1}$ Department of Pathology, Brain Korea 21 PLUS Project for Medical Science, Integrated Genomic Research \\ Center for Metabolic Regulation, Yonsei University College of Medicine, Seoul, Korea; ${ }^{2}$ Department of \\ Pathology, Seoul National University Bundang Hospital, Seoul National University College of Medicine, \\ Seongnam, Korea; ${ }^{3}$ Department of Surgery, Yonsei University College of Medicine, Seoul, Korea; ${ }^{4}$ Biostatistics \\ Collaboration Unit, Department of Research Affairs, Yonsei University College of Medicine, Seoul, Korea and \\ ${ }^{5}$ Severance Biomedical Science Institute, Yonsei University College of Medicine, Seoul, Korea
}

Hypoxia is known to be important in the generation and maintenance of stemness; however, its clinical significance is yet to be determined in human hepatocellular carcinoma. The expression of stemness (K19, EpCAM) and hypoxia (carbonic anhydrase-IX (CAIX))-related markers were investigated by immunohistochemistry in three hepatocellular carcinoma cohorts. The clinicopathologic features, response to transarterial chemoembolization, and outcomes were compared. In cohort $1(n=14$, biopsy-transarterial chemoembolizationresection-matched hepatocellular carcinoma), all K19-, EpCAM-, or CAIX-positive hepatocellular carcinomas on initial biopsy $(6 / 6,100 \%)$ showed residual tumors after transarterial chemoembolization, whereas $75 \%(6 / 8)$ of allnegative hepatocellular carcinomas on biopsy showed complete necrosis in the post-transarterial chemoembolization-resected specimens. In cohort $2(n=85$, explanted hepatocellular carcinomas with/without transarterial chemoembolization; totally necrotic hepatocellular carcinoma after transarterial chemoembolization was not included), the expression of K19, EpCAM, and CAIX, and their coexpression, was more frequently observed with a greater number of transarterial chemoembolization sessions, and the expression of these markers was also correlated to each other. CAIX expression was shown to be an independent factor for recurrence and survival, and combination of CAIX with Milan criteria significantly increased the time-dependent integrative area under the curve values for recurrence and survival. In cohort $3(n=339$, resected hepatocellular carcinomas without transarterial chemoembolization), CAIX(+) hepatocellular carcinomas exhibited higher K19 and EpCAM expression, and more invasive pathological features. CAIX expression and TNM stage were independent predictors of extrahepatic recurrence, and the addition of CAIX to the TNM stage significantly increased timedependent integrative area under the curve values. In conclusion, the expression of stemness (K19, EpCAM) and hypoxia (CAIX)-related markers were correlated each other, and hepatocellular carcinoma expressing these markers showed resistance to transarterial chemoembolization and poorer outcome. Evaluation for both markers of stemness and hypoxia may have an additional value in predicting hepatocellular carcinoma outcome, especially for transarterial chemoembolization-treated hepatocellular carcinomas.

Modern Pathology (2016) 29, 1038-1049; doi:10.1038/modpathol.2016.111; published online 17 June 2016

Correspondence: Professor YN Park, MD, PhD, Department of Pathology, Brain Korea 21 PLUS Project for Medical Science, Integrated Genomic Research Center for Metabolic Regulation, Yonsei University College of Medicine, CPO Box 8044, 250 Seongsanno, Seodaemun-gu, Seoul 120-752, Korea.

E-mail: young0608@yuhs.ac

Received 22 January 2016; revised 6 May 2016; accepted 6 May 2016; published online 17 June 2016
Hypoxia, which is observed in most solid tumors because of their rapid growth and a relative lack of blood supply, is known to contribute to the progression and biological aggressiveness of tumors. ${ }^{1}$ Tumor cells adapt to the unfavorable hypoxic microenvironment by expressing hypoxiainducible factors, including HIF1 $\alpha$, which in turn 
activate target genes involved in proliferation, angiogenesis, and epithelial-mesenchymal transition, resulting in a more malignant phenotype. ${ }^{2}$ Carbonic anhydrase-IX (CAIX), a HIF1 $\alpha$-regulated $\mathrm{pH}$-modifying enzyme, also contributes to cell survival, migration, and invasion in hypoxic microenvironments. ${ }^{3}$ Hypoxia has also been reported to be important in reprogramming to cancer stem cell phenotype and maintenance of cancer stem cells in several cancer types, including hepatocellular carcinoma, glioblastoma, breast cancer, and hematologic malignancies. ${ }^{4-7}$ Cancer stem cells, characterized by their ability to self-renew and propagate tumors, have an important role in tumor maintenance and recurrence. ${ }^{8}$ We recently reported that hepatocellular carcinomas expressing stemnessrelated markers, such as keratin 19 (K19) and epithelial cell adhesion molecule (EpCAM), are associated with epithelial-mesenchymal transition phenotype and a poorer prognosis compared with hepatocellular carcinomas that do not express these markers. ${ }^{9,10}$

Hepatic resection and transplantation are the treatments of choice in early-stage hepatocellular carcinoma patients; however, they are only indicated in a small proportion of hepatocellular carcinoma patients. ${ }^{11,12}$ To reduce tumor burden and to prevent dropout from transplantation waiting lists, various locoregional therapies have been applied to hepatocellular carcinoma before resection or transplantation. ${ }^{13}$ Transarterial chemoembolization is one of the most widely used locoregional therapies, and it utilizes severe hypoxia resulting from hepatic arterial embolization and cytotoxic agents to eliminate tumor cells. However, the response to transarterial chemoembolization varies, and a significant number of hepatocellular carcinomas (50-86\%) show residual viable tumor. ${ }^{14}$

Despite the biological significance of hypoxic microenvironment in many solid tumors, the clinical significance of hypoxic microenvironment in hepatocellular carcinoma is not well described yet. Therefore, we addressed the associations between the expression of hypoxia- and stemness-related markers, the clinicopathologic parameters, response to transarterial chemoembolization, and patients' outcome in three independent cohorts of biopsyresection-matched, explanted, or resected hepatocellular carcinomas with or without preoperative transarterial chemoembolization.

\section{Materials and methods}

\section{Human Hepatocellular Carcinoma Tissue Samples and Clinicopathologic Analysis}

This study was performed on hepatocellular carcinoma tissue samples taken from three cohorts. The hepatocellular carcinomas included in this study were morphologically typical; cases that could be classified as combined hepatocellular cholangiocarcinoma by hematoxylin-eosin or mucin stains were excluded. The clinicopathologic characteristics of the three hepatocellular carcinoma cohorts are summarized in Supplementary Table 1. This study was approved by the institutional review board of Severance Hospital (4-2012-0755).

Cohort 1 consisted of 14 cases of biopsytransarterial chemoembolization-resection-matched hepatocellular carcinomas. All the 14 cases were initially diagnosed with hepatocellular carcinoma on biopsy, followed by one session of transarterial chemoembolization, and subsequently underwent curative resection between January 2001 and December 2008 at Severance Hospital, Yonsei University Medical Center. Only single nodular hepatocellular carcinomas were enrolled to ensure that the same nodule was biopsied and treated with transarterial chemoembolization. The interval between biopsy and resection after one session of transarterial chemoembolization was less than 90 days.

Cohort 2 comprised hepatocellular carcinoma patients who underwent liver transplantation at the same institution between December 2001 and February 2013. One hundred sixty-six cases of transplanted hepatocellular carcinoma were enrolled, and 81 patients were excluded for the following reasons: (a) no remaining viable tumor lesion due to extensive necrosis induced by transarterial chemoembolization $(n=69)$, (b) history of systemic chemotherapy $(n=6)$, or (c) only treated with locoregional therapies other than transarterial chemoembolization $(n=6)$. Finally, 85 patients were included in cohort 2, and representative paraffin-embedded sections with residual hepatocellular carcinoma were used for immunohistochemical staining. Among 85 cases of cohort $2,34(40 \%)$ were not subjected to any locoregional therapy, whereas $51(60 \%)$ hepatocellular carcinomas were subjected to at least one session of transarterial chemoembolization (median two sessions, interquartile range 1-4 sessions, range 1-14 sessions), and 17 cases also underwent other locoregional therapies (radiofrequency ablation, ${ }^{166}$ holmium injection, or radiation therapy; median one session, interquartile range 1-3 sessions, range 1-3 sessions) in addition to transarterial chemoembolization.

Cohort 3 consisted of 339 consecutive hepatocellular carcinoma patients who did not undergo any kind of preoperative treatment before hepatic resection. These consecutive hepatocellular carcinoma samples were obtained by hepatic resection between March 2006 and February 2011 at the same institution. The representative paraffin-embedded sections of hepatocellular carcinoma were used for tissue microarray construction and immunohistochemical analysis.

Immunohistochemistry was performed for CAIX (1:1000; Abcam, Cambridge, MA, USA), K19 (1:100; DAKO, Glostrup, Denmark), and EpCAM (1:1000; 
Calbiochem, Darmstadt, Germany) on representative sections of whole paraffin-embedded tissues from cohorts 1 and 2, and tissue microarray sections from cohort 3. For resected/explanted cases after transarterial chemoembolization, representative sections containing residual hepatocellular carcinoma was immunostained.

For more detailed information on immunohistochemistry, tissue microarray construction, and measurement of outcome, please refer to the Supplementary Materials and Methods.

\section{Statistical Methods}

Statistical analysis was conducted using SPSS (version 20.0.1, SPSS, Chicago, IL, USA) or R (version 3.1.1, http://www.r-project.org/) software. Mann-Whitney $U$-test, $\chi^{2}$-test, or Fisher's exact test were used as deemed appropriate. Survival analyses were performed by the Kaplan-Meier method, log-rank test, or Cox regression analysis. Statistical significance was assumed when $P<0.05$. For timedependent integrative area under the curve analysis, package risksetROC was used, and a bootstrappingbased method was conducted to estimate differences in integrative area under the curve values.

\section{Results}

The Expression of CAIX, K19, and EpCAM, and

Treatment Response to Transarterial

Chemoembolization in Biopsy-Transarterial

Chemoembolization-Resection-Matched

Hepatocellular Carcinomas (Cohort 1, $n=14$ )

To assess the response of hepatocellular carcinoma to transarterial chemoembolization according to the expression status of stemness- or hypoxia-related markers, we enrolled 14 patients who underwent biopsy, one session of transarterial chemoembolization, and subsequent curative resection for single nodular hepatocellular carcinoma. Most (64\%) hepatocellular carcinomas were hepatitis B virus-related (Supplementary Table 1). The response to transarterial chemoembolization was evaluated by the proportion of residual tumor in the resected specimen.

In the initial (pre-transarterial chemoembolization) biopsies, CAIX, K19, and EpCAM expression was seen in $14 \%(2 / 14), 21 \%(3 / 14)$, and $36 \%(5 / 14)$, respectively (Figures $1 \mathrm{a}$ and $\mathrm{b}$ ), and all of CAIXpositive hepatocellular carcinomas on biopsies were also positive for K19 and EpCAM. In the subsequently resected (post-transarterial chemoembolization) hepatocellular carcinomas, the expression of CAIX, K19, and EpCAM was similar to that of the matched biopsies; case numbers 1, 2, 4, 5, and 8 showed the same immunohistochemical profiles, and case numbers 3,6 , and 7 showed different expression in only one marker. Hepatocellular carcinomas showing negativity for all three markers in the biopsy $(8 / 14,57 \%)$ frequently demonstrated total tumor necrosis $(6 / 8,75 \%)$ in the resected posttransarterial chemoembolization specimens. In contrast, all hepatocellular carcinomas expressing at least one of K19, EpCAM, or CAIX in the biopsies (6/14, 43\%) revealed residual hepatocellular carcinomas in the matched resected livers after transarterial chemoembolization, and the proportion of the residual tumor ranged from 5 to $90 \%$ (Figure 1c and Supplementary Table 2). In addition, deaths within 5 years $(n=3)$ and extrahepatic recurrence within 2 years $(n=3)$ were only observed in hepatocellular carcinomas showing positive expression of K19, EpCAM, or CAIX. Furthermore, two out of three metastasized and deceased cases were triple-positive for K19, EpCAM, and CAIX.

\section{The Expression of K19, EpCAM, and CAIX and Patient's Outcome in Explanted Patients With/Without Transarterial Chemoembolization (Cohort 2, $n=85$ )}

To further assess the clinical effect of hypoxic microenvironment on hepatocellular carcinomas with stemness-related markers, we enrolled 85 explanted hepatocellular carcinomas with/without a history of transarterial chemoembolization, most of which $(82 \%)$ were hepatitis B virus-related (Supplementary Table 1). The cases with total necrotic tumor after transarterial chemoembolization were not included as immunohistochemistry could not be applicable. Thirty-four (40\%) cases were not subjected to any preoperative locoregional therapy, whereas $51(60 \%)$ hepatocellular carcinomas were subjected to at least one session of transarterial chemoembolization (median two sessions, interquartile range 1-4 sessions, range 1-14 sessions).

The protein expression levels of K19, EpCAM, and CAIX were compared among hepatocellular carcinomas without transarterial chemoembolization, those treated with one to three sessions of transarterial chemoembolization, and those treated with four or more sessions of transarterial chemoembolization. Interestingly, the incidence of K19, EpCAM, and CAIX positivity and coexpression of these markers were significantly increased as the number of transarterial chemoembolization sessions increased (Figures 2a and b, $P<0.05$ for all). In addition, there were significant positive correlations between CAIX and K19 $(P=0.001)$, CAIX and EpCAM $(P=0.048)$, as well as between K19 and EpCAM $(P<0.001$, Figure 2c and Supplementary Figure 1A). The clinicopathologic features of the explanted hepatocellular carcinomas in preoperative-transarterial chemoembolization and non-preoperative-transarterial chemoembolization groups are summarized in Supplementary Table 3 . The cases with transarterial chemoembolization showed higher serum aspartate transaminase and alanine transaminase levels, more frequent tumor encapsulation, and K19 expression 
a

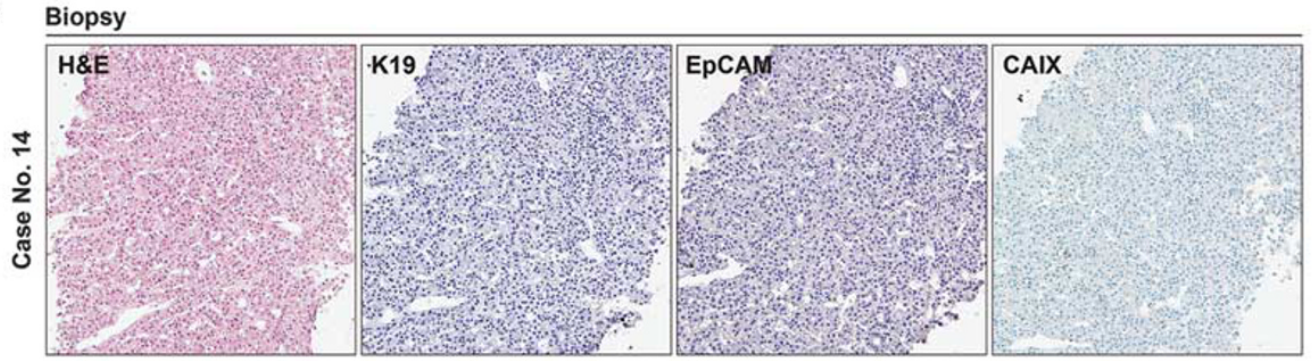

Resection

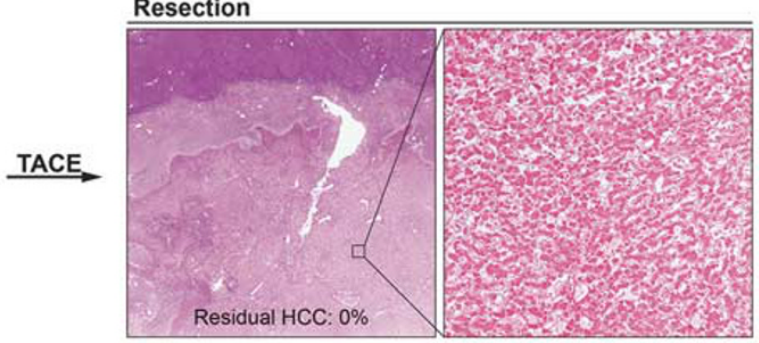

\section{Biopsy}
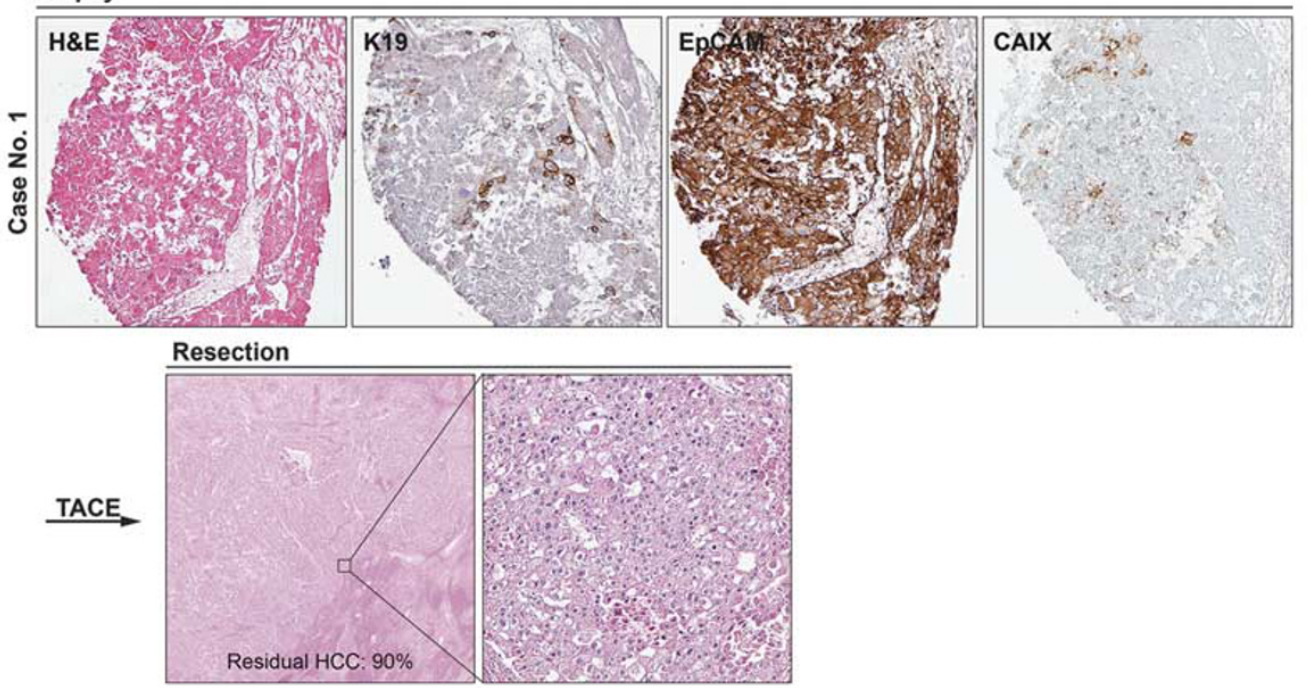

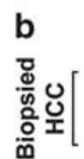

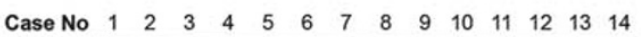
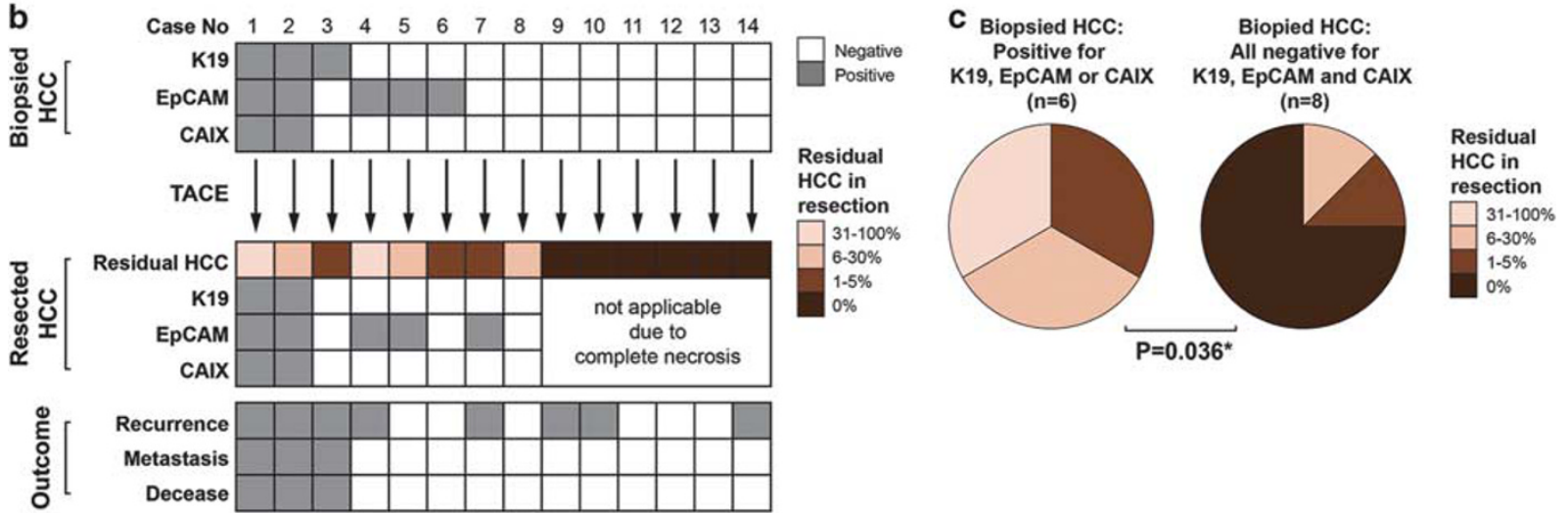

Figure 1 Expression of K19, epithelial cell adhesion molecule (EpCAM), and carbonic anhydrase-IX (CAIX) in HCCs and the response to TACC (cohort 1, $n=14$ ). (a) Representative cases of K19-, EpCAM-, and CAIX-negative HCC and K19-, EpCAM-, and CAIX-positive HCC and the proportion of residual tumor in resected specimens after TACC. (b) The expression status of K19, EpCAM, and CAIX, response to TACC, and outcome in individual HCC patients with biopsy-resection-matched tissue. The outcome was measured as recurrence within 2 years, extrahepatic recurrence within 2 years, and death within 5 years. (c) Comparison of TACC response between HCCs expressing at least one of K19, EpCAM, or CAIX $(n=6)$ and HCCs expressing none of three markers $(n=8)$ in biopsy. ${ }^{*} P<0.05$. HCC, hepatocellular carcinoma; TACE, transarterial chemoembolization. 


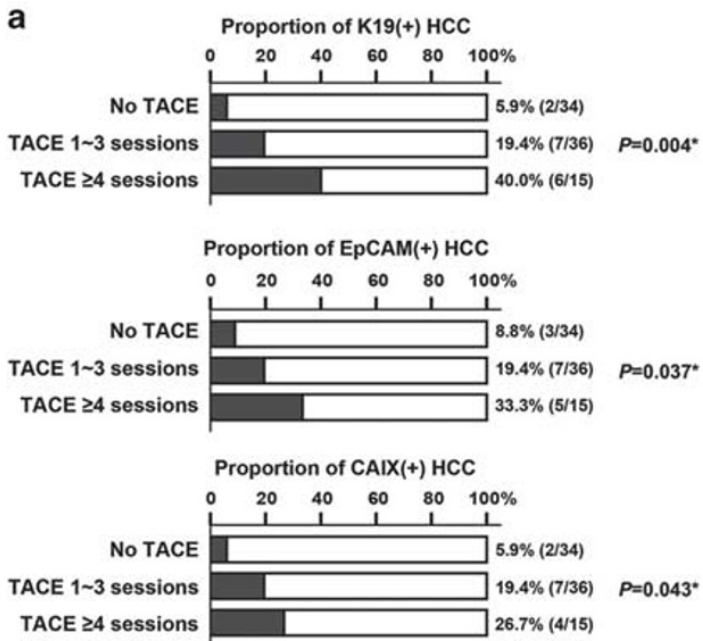

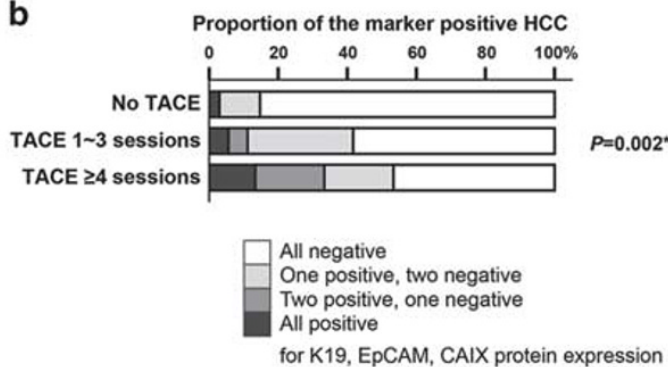

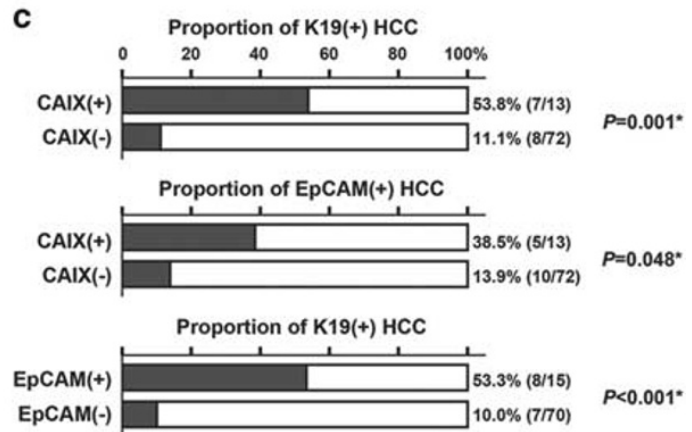

d

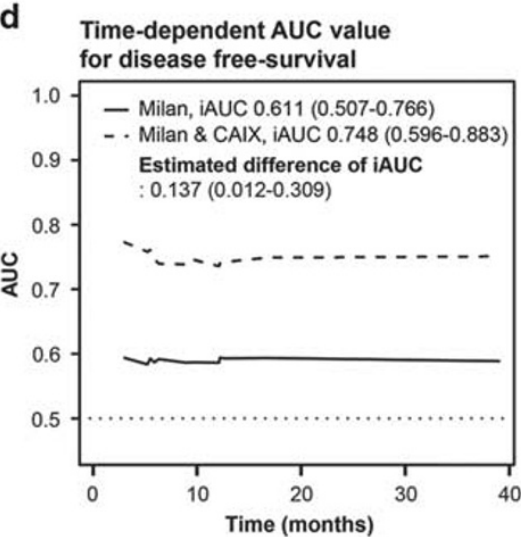

f

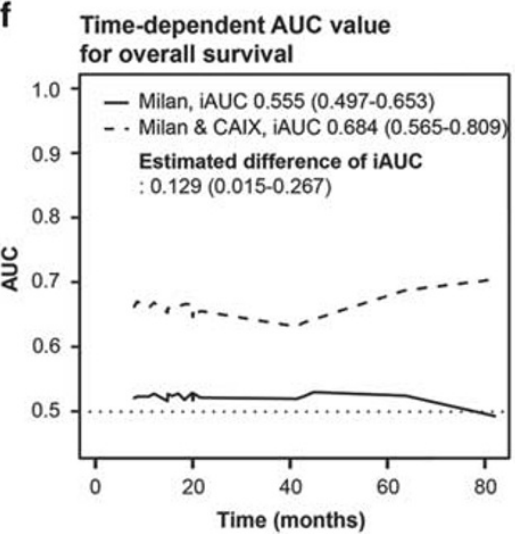

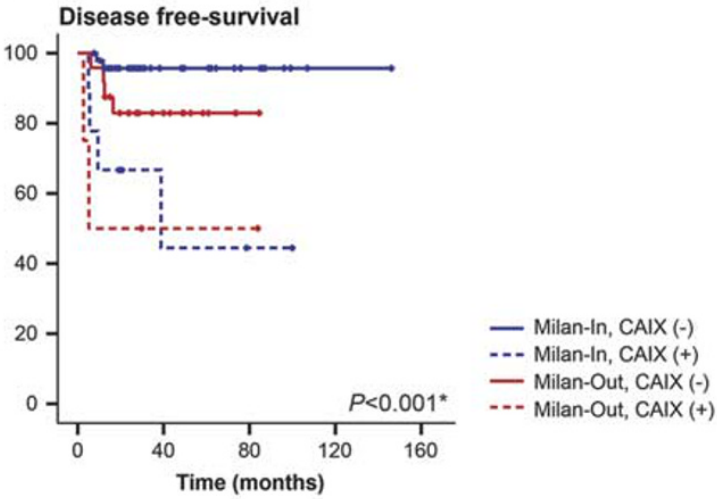

g

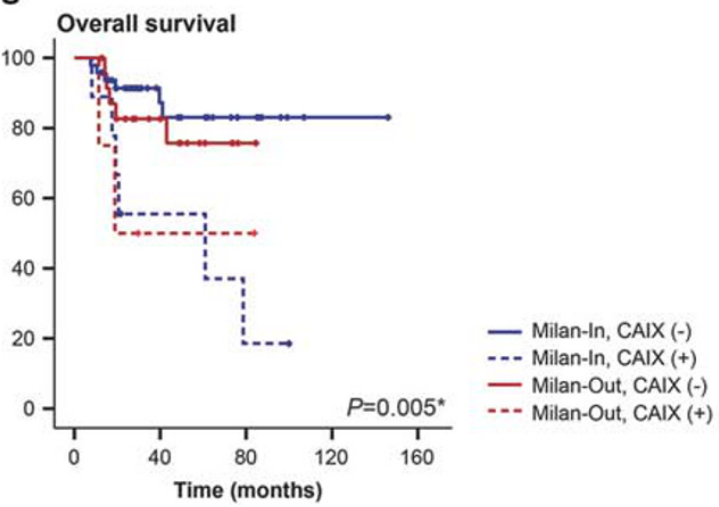

Figure 2 Expression of K19, epithelial cell adhesion molecule (EpCAM), and carbonic anhydrase-IX (CAIX) and patients' outcome in explanted HCCs with/without TACC (cohort 2, $n=85$ ). (a, b) The expression status of K19, EpCAM, and CAIX proteins according to the number of TACC sessions. (c) Correlations between K19, EpCAM, and CAIX protein expression. (d) Time-dependent iAUC analysis for recurrence in comparison between Milan criteria and Milan criteria combined with CAIX protein expression. (e) Kaplan-Meier plot for disease-free survival according to the Milan criteria and CAIX protein expression. (f) Time-dependent iAUC analysis for overall survival in comparison between Milan criteria and Milan criteria combined with CAIX protein expression. (g) Kaplan-Meier plot for overall survival according to Milan criteria and CAIX protein expression. ${ }^{*} P<0.05$. HCC, hepatocellular carcinoma; iAUC, integrative area under the curve; TACE, transarterial chemoembolization. 
Table 1 Clinicopathological features of hepatocellular carcinomas in cohort 2 according to the CAIX protein expression status

\begin{tabular}{|c|c|c|c|}
\hline Clinicopathologic features & CAIX positive $\mathrm{n}=13(15 \%)$ & CAIX negative $\mathrm{n}=72(85 \%)$ & P-value \\
\hline Age (year) & $52(45-56)$ & $54(50-60)$ & 0.067 \\
\hline Gender (male/female) & $10(77 \%) / 3(23 \%)$ & $59(82 \%) / 13(18 \%)$ & 0.670 \\
\hline Etiology (hepatitis B/hepatitis C/alcohol/unknown) & $11(85 \%) / 1(8 \%) / 1(8 \%) / 0(0 \%)$ & $59(82 \%) / 8(11 \%) / 4(6 \%) / 1(1 \%)$ & 0.941 \\
\hline Cirrhosis & $13(100 \%)$ & $70(97 \%)$ & 1.000 \\
\hline Serum aspartate transaminase (IU/l) & $42(35-75)$ & $46(35-68)$ & 0.779 \\
\hline Serum alanine transaminase (IU/l) & $31(13-58)$ & $32(20-40)$ & 0.883 \\
\hline Serum albumin (g/dl) & $3.0(2.6-3.8)$ & $3.3(2.8-3.6)$ & 0.399 \\
\hline Serum platelet $(1000 / \mu \mathrm{l})$ & $66(41-101)$ & $79(54-118)$ & 0.173 \\
\hline Serum alpha-fetoprotein (IU/ml) & $29.0(2.9-246.2)$ & $10.9(4.8-60.9)$ & 0.502 \\
\hline Serum PIVKA-II $(\mathrm{AU} / \mathrm{ml})^{\mathrm{a}}$ & $76.0(24.0-402.3)$ & $37.5(18.3-96.0)$ & 0.131 \\
\hline \multicolumn{4}{|l|}{ Tumor pathology } \\
\hline Diameter of largest tumor $(\mathrm{cm})$ & $3.0(2.3-3.5)$ & $2.2(1.6-3.5)$ & 0.319 \\
\hline Total tumor diameter $(\mathrm{cm})$ & $3.5(2.4-5.5)$ & $3.6(2.4-6.4)$ & 0.665 \\
\hline Differentiation (Edmonson-Steiner grades I/II/III) & $1(8 \%) / 11(85 \%) / 1(8 \%)$ & $27(38 \%) / 40(56 \%) / 5(7 \%)$ & 0.104 \\
\hline Capsule formation (absent/partial/complete) & $0(0 \%) / 9(69 \%) / 4(31 \%)$ & $22(31 \%) / 32(44 \%) / 18(25 \%)$ & 0.063 \\
\hline Microvascular invasion & $8(62 \%)$ & $13(18 \%)$ & $0.002^{\mathrm{b}}$ \\
\hline Number of the lesion $(1 / 2$ or $3 / \geq 4)$ & $6(46 \%) / 6(46 \%) / 1(8 \%)$ & $23(32 \%) / 35(49 \%) / 14(19 \%)$ & 0.466 \\
\hline Milan status (in/out) & $9(69 \%) / 4(31 \%)$ & $48(67 \%) / 24(33 \%)$ & 1.000 \\
\hline K19 protein expression & $7(54 \%)$ & $8(11 \%)$ & $0.001^{\mathrm{b}}$ \\
\hline EpCAM protein expression & $5(39 \%)$ & $10(14 \%)$ & $0.048^{\mathrm{b}}$ \\
\hline
\end{tabular}

Variables are expressed as median (interquartile range) or number (\%).

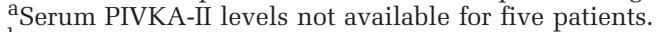

bindicates $P<0.05$.

Table 2 Univariate and multivariate analyses of disease-free survival in cohort 2

\begin{tabular}{|c|c|c|c|c|}
\hline \multirow{2}{*}{ Variables } & \multicolumn{2}{|l|}{ Univariate analysis } & \multicolumn{2}{|c|}{ Multivariate analysis } \\
\hline & $\begin{array}{c}\text { Hazard ratio ( } 95 \% \text { confidence } \\
\text { interval) }\end{array}$ & P-value ${ }^{a}$ & $\begin{array}{l}\text { Hazard ratio ( } 95 \% \\
\text { confidence interval) }\end{array}$ & $\mathrm{P}$-value $\mathrm{a}^{\mathrm{a}}$ \\
\hline Age ( $\geq 60$ years) & $0.03(0.0-8.1)$ & 0.222 & & \\
\hline Gender (male) & $0.8(0.2-2.8)$ & 0.677 & & \\
\hline Etiology (hepatitis B virus) & $2.4(0.3-18.7)$ & 0.400 & & \\
\hline Serum alpha-fetoprotein $(\geq 400 \mathrm{IU} / \mathrm{ml})$ & $8.4(2.6-26.6)$ & $<0.001^{\mathrm{b}}$ & $6.8(1.8-26.1)$ & $0.005^{b}$ \\
\hline Serum PIVKA-II $(\geq 400 \mathrm{AU} / \mathrm{ml})$ & $6.0(1.8-20.0)$ & $0.004^{\mathrm{b}}$ & $2.0(0.5-8.7)$ & 0.358 \\
\hline Microvascular invasion (positive) & $2.4(0.8-7.5)$ & 0.138 & & \\
\hline Differentiation (Edmonson-Steiner grade III) & $1.4(0.2-10.6)$ & 0.769 & & \\
\hline Milan status (out of Milan) & $2.1(0.7-6.4)$ & 0.209 & & \\
\hline $\begin{array}{l}\text { Pre-transplantation transarterial } \\
\text { chemoembolization (one or more sessions) }\end{array}$ & $7.7(1.0-59.5)$ & 0.051 & & \\
\hline CAIX protein expression (positive) & $7.4(2.4-22.9)$ & $0.001^{\mathrm{b}}$ & $6.4(1.9-22.0)$ & $0.003^{b}$ \\
\hline K19 protein expression (positive) ${ }^{\mathrm{C}}$ & $3.5(1.4-8.7)$ & $0.007^{\mathrm{b}}$ & & \\
\hline EpCAM protein expression (positive) ${ }^{\mathrm{C}}$ & $3.6(1.4-8.8)$ & $0.006^{\mathrm{b}}$ & & \\
\hline
\end{tabular}

${ }^{a}$ Univariate and multivariate analyses were performed using the Cox proportional hazards model.

bindicates $P<0.05$.

${ }^{\mathrm{C}} \mathrm{K} 19$ and EpCAM were not included in the multivariate analysis because of significant associations with CAIX.

$(P<0.05$ for all), and there were no significant differences in the other clinicopathological features.

According to the CAIX protein expression status, CAIX-positive hepatocellular carcinomas showed more frequent microvascular invasion and higher expression of K19 or EpCAM compared with CAIXnegative hepatocellular carcinomas $(P<0.05$ for all, Table 1). There were no significant differences in the other clinicopathological features according to the CAIX expression status, including Milan status, size, and differentiation of hepatocellular carcinomas.
With regard to hepatocellular carcinoma patient outcome, high serum alpha-fetoprotein levels $(\geq 400 \mathrm{IU} / \mathrm{ml})$, high serum PIVKA-II $(\geq 400 \mathrm{AU} / \mathrm{ml})$, and K19, EpCAM, and CAIX protein expression were significantly associated with a poor disease-free survival $(P<0.05$ for all, Table 2, Supplementary Figure 1B). As K19, EpCAM, and CAIX were significantly correlated to each other, only CAIX, the most predictive marker, was included for further multivariate analysis (Supplementary Figure 1D). High serum alpha-fetoprotein level and CAIX protein 
Table 3 Univariate and multivariate analyses of overall survival in cohort 2

\begin{tabular}{|c|c|c|c|c|}
\hline \multirow{2}{*}{ Variables } & \multicolumn{2}{|l|}{ Univariate analysis } & \multicolumn{2}{|c|}{ Multivariate analysis } \\
\hline & $\begin{array}{l}\text { Hazard ratio (95\% confidence } \\
\text { interval) }\end{array}$ & $\mathrm{P}$-value & $\begin{array}{l}\text { Hazard ratio (95\% } \\
\text { confidence interval) }\end{array}$ & $\mathrm{P}$-value $\mathrm{a}^{\mathrm{a}}$ \\
\hline Age ( $\geq 60$ years) & $1.2(0.4-3.4)$ & 0.705 & & \\
\hline Gender (male) & $1.3(0.5-3.7)$ & 0.594 & & \\
\hline Etiology (hepatitis B virus) & $0.6(0.2-1.6)$ & 0.302 & & \\
\hline Serum alpha-fetoprotein $(\geq 400 \mathrm{IU} / \mathrm{ml})$ & $2.0(0.6-6.9)$ & 0.276 & & \\
\hline Serum PIVKA-II $(\geq 400 \mathrm{AU} / \mathrm{ml})$ & $1.3(0.3-5.8)$ & 0.710 & & \\
\hline Microvascular invasion (positive) & $3.6(1.4-8.9)$ & $0.006^{\mathrm{b}}$ & $2.2(0.7-6.5)$ & 0.735 \\
\hline Differentiation (Edmonson-Steiner grade III) & $1.3(0.8-2.1)$ & 0.354 & & \\
\hline Milan status (out of Milan) & $1.2(0.5-3.1)$ & 0.685 & & \\
\hline $\begin{array}{l}\text { Pre-transplantation transarterial chemoembolization } \\
\text { (one or more sessions) }\end{array}$ & $2.4(0.8-7.1)$ & 0.128 & & \\
\hline CAIX protein expression (positive) & $4.5(1.8-11.2)$ & $0.001^{\mathrm{b}}$ & $2.9(1.0-8.6)$ & 0.062 \\
\hline K19 protein expression (positive) ${ }^{\mathrm{C}}$ & $2.5(1.0-6.1)$ & $0.040^{\mathrm{b}}$ & & \\
\hline EpCAM protein expression (positive) ${ }^{\mathrm{C}}$ & $2.3(0.9-5.4)$ & 0.072 & & \\
\hline
\end{tabular}

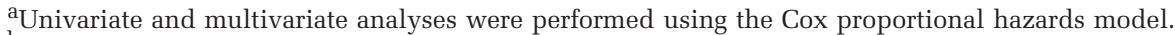

${ }^{\mathrm{b}}$ Indicates $P<0.05$.

${ }^{\mathrm{C}} \mathrm{K} 19$ and EpCAM were not included in the multivariate analysis because of significant associations with CAIX.

expression remained as independent prognostic factors for recurrence $(P=0.005, P=0.003$, respectively). Regarding overall survival, CAIX and K19 protein expression and microvascular invasion were significant in univariate analysis $(P=0.001$, $P=0.040, \quad P=0.006$, respectively, Table 3 and Supplementary Figure 1C). As in the analysis of disease-free survival, only CAIX protein expression was chosen for inclusion in multivariate analysis (Supplementary Figure 1E), and it showed borderline significance $(P=0.062)$.

Among the 85 transplantation patients in cohort 2, 57 cases (67\%) fulfilled the Milan criteria (Milan-In), whereas $28(33 \%)$ did not (Milan-Out). Combining CAIX expression and Milan status yielded higher integrative area under the curve values for predicting recurrence and overall survival compared with integrative area under the curve values for the Milan status alone (estimated differences: 0.137 (95\% confidence interval, 0.012-0.309) for recurrence, 0.129 (95\% confidence interval, 0.015-0.267) for overall survival). The CAIX-negative/Milan-In patients demonstrated the best outcomes compared with other groups, and, interestingly, CAIX-negative/ Milan-Out patients demonstrated more favorable outcomes than CAIX-positive/Milan-In or Out patients (Figure 2d-g). The Milan status was determined regardless of whether the hepatocellular carcinomas were early or progressed hepatocellular carcinomas; however, the prognosis of early hepatocellular carcinoma is excellent when properly treated. ${ }^{15,16}$ In cohort 2, 32 of the 85 patients $(38 \%)$ had one or more early hepatocellular carcinomas on pathological examination of explanted livers, and, therefore, 7 of $28(25 \%)$ patients initially classified as Milan-Out could have fulfilled the Milan criteria. Thus, we analyzed prognoses according to the Milan criteria after excluding all early hepatocellular carcinomas. Therein, Milan criteria were shown to be a significant prognostic factor for recurrence, and CAIX-negative/Milan-In patients still showed the best outcomes for recurrence and survival (Supplementary Figure 2A, B).

\section{The Expression of K19, EpCAM, and CAIX, and Patient's Outcome in Resected Hepatocellular Carcinomas Without Preoperative Transarterial Chemoembolization (Cohort 3, $n=339$ )}

We further investigated the relationship between hypoxia markers and stemness-related markers, and compared the clinicopathologic features according to the expression status of these markers in resected hepatocellular carcinomas without any preoperative treatment (cohort 3, $n=339$; Supplementary Table 1). The majority $(82 \%)$ of these hepatocellular carcinomas were hepatitis B virus-related.

Immunohistochemical stains on the tissue microarray demonstrated 35 (10\%) K19-positive, 117 (35\%) EpCAM-positive, and 34 (10\%) CAIXpositive hepatocellular carcinomas (Figure 3a and Supplementary Figure 3A). K19 or EpCAM expression was more frequently seen in CAIX-positive hepatocellular carcinomas $(P<0.001, \quad P=0.004$, respectively, Figure 3b). EpCAM-positive hepatocellular carcinomas were also more frequently positive for K19 $(P<0.001)$.

Table 4 lists the clinicopathological features of cohort 3 according to the expression status of CAIX protein. CAIX-positive hepatocellular carcinomas were more frequently associated with high serum alpha-fetoprotein $(P<0.001)$ and PIVKA-II levels $(P<0.001)$, poorer differentiation $(P=0.002)$, microvascular invasion $(P=0.002)$, and advanced TNM stage $(P=0.024)$ compared with CAIX-negative hepatocellular carcinomas. To address the topographical 
a

a Cohort 3

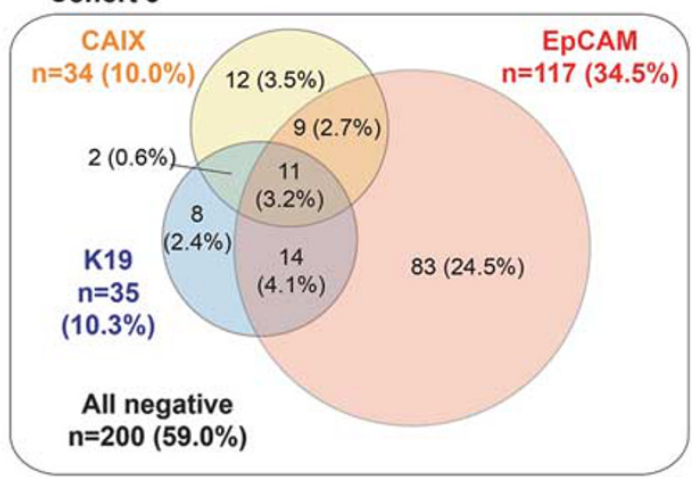

C
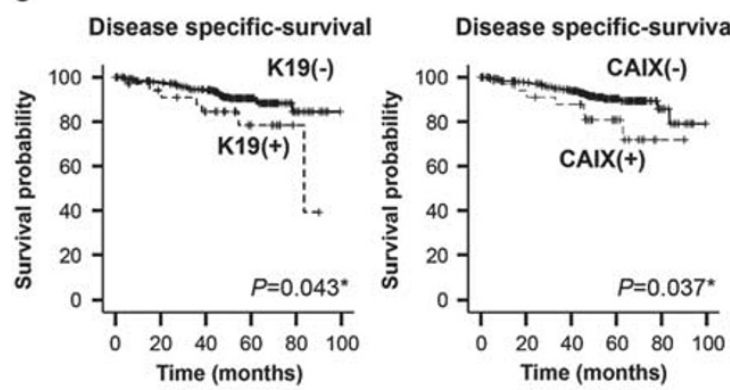

b

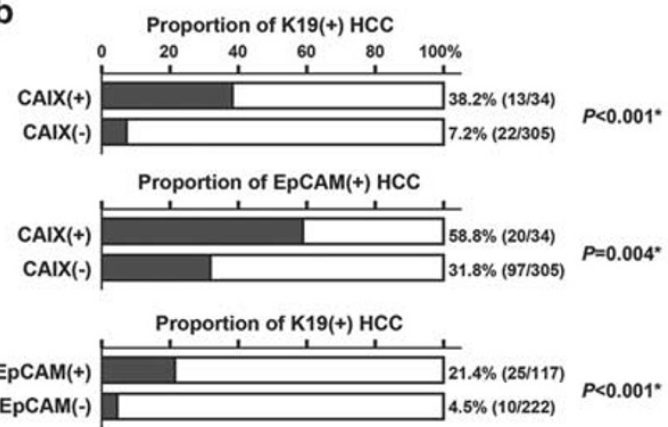

d Time-dependent AUC value for extrahepatic recurrence-free survival

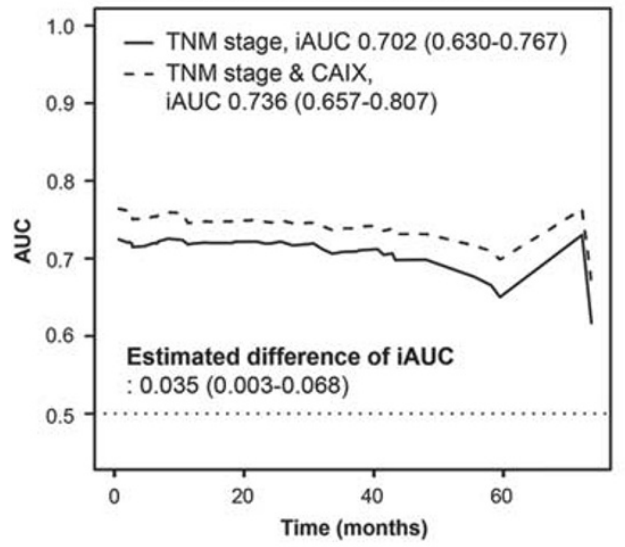

Extrahepatic recurrencefree survival

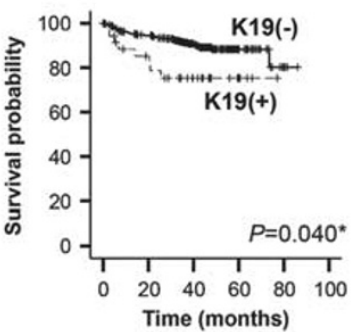

Extrahepatic recurrencefree survival

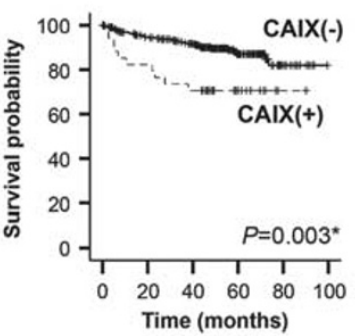

e

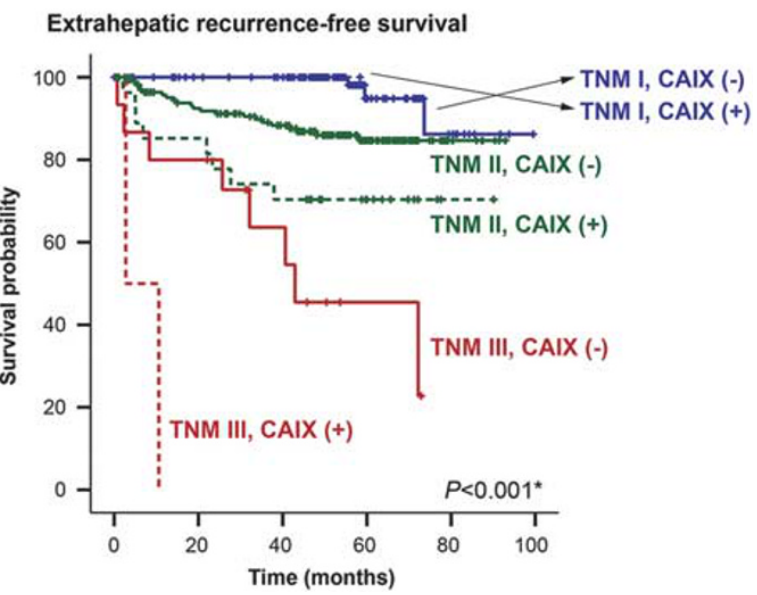

Figure 3 Expression of K19, epithelial cell adhesion molecule (EpCAM), and carbonic anhydrase-IX (CAIX), and their value in predicting outcome in resected HCCs (cohort 3, $n=339$ ). Venn diagram (a) and bar graphs (b) demonstrating the correlation between K19, EpCAM, and CAIX expression in HCCs. (c) Survival curves showing decreased disease-specific survival or extrahepatic recurrence-free survival in K19-positive and CAIX-positive HCCs. (d) Time-dependent iAUC analysis for extrahepatic recurrence, showing the increased iAUC value for TNM stage combined with the CAIX status in comparison with the TNM stage alone. (e) Kaplan-Meier plot of extrahepatic recurrencefree survival according to the TNM stage and CAIX expression status. ${ }^{*} P<0.05$. HCC, hepatocellular carcinoma; iAUC, integrative area under the curve.

correlation between CAIX expression and necrosis, we compared the presence of necrosis and CAIX expression in the tissue microarray cores. CAIX expression was more frequently seen in the tissue microarray cores with necrosis compared with that without necrosis $(P<0.001)$.

K19 and CAIX expression levels were associated with significantly shorter disease-specific survival (log-rank, $P=0.043, P=0.037$, respectively), and also shorter extrahepatic recurrence-free survival (log-rank, $P=0.040, P=0.003$, respectively, Figure 3c). No significant differences were seen for diseasespecific survival or extrahepatic recurrence-free survival according to the EpCAM expression status, and disease-free and overall survival were not significantly different according to K19, EpCAM, or CAIX status (Supplementary Figure 3B-D).

On univariate analysis using a Cox proportional hazards model, high serum PIVKA-II ( $\geq 400 \mathrm{AU} / \mathrm{ml}$ ), poor differentiation, TNM stage, and K19 and CAIX 
Table 4 Clinicopathological features of hepatocellular carcinomas in cohort 3 according to the CAIX protein expression status

\begin{tabular}{|c|c|c|c|}
\hline Clinicopathologic features & CAIX positive $\mathrm{n}=34(10 \%)$ & CAIX negative $\mathrm{n}=305(90 \%)$ & $\mathrm{P}$-value \\
\hline Age (year) & $51(46-64)$ & $56(48-63)$ & 0.400 \\
\hline Gender (male/female) & $25(74 \%) / 9(27 \%)$ & $252(83 \%) / 53(17 \%)$ & 0.239 \\
\hline Etiology (hepatitis B/hepatitis C/alcohol/unknown) & $28(82 \%) / 1(3 \%) / 1(3 \%) / 4(12 \%)$ & $249(82 \%) / 17(6 \%) / 14(5 \%) / 25(8 \%)$ & 0.791 \\
\hline Cirrhosis & $17(50 \%)$ & $170(56 \%)$ & 0.587 \\
\hline Serum aspartate transaminase (IU/l) & $30(24-39)$ & $30(24-41)$ & 0.816 \\
\hline Serum alanine transaminase (IU/l) & $29(19-40)$ & $31(22-46)$ & 0.327 \\
\hline Serum albumin (g/dl) & $4.4(4.1-4.7)$ & $4.4(4.1-4.7)$ & 0.882 \\
\hline Serum platelet $(1000 / \mu \mathrm{l})$ & 164 (124-209) & $163(132-208)$ & 0.962 \\
\hline Serum alpha-fetoprotein $(\mathrm{IU} / \mathrm{ml})^{\mathrm{a}}$ & $194.9(20.8-1315.5)$ & $17.8(3.8-183.6)$ & $<0.001^{\mathrm{b}}$ \\
\hline Serum PIVKA-II $(\mathrm{AU} / \mathrm{ml})^{\mathrm{C}}$ & $606.0(117.0-1870.0)$ & $72.0(30.0-372.0)$ & $<0.001^{\mathrm{b}}$ \\
\hline \multicolumn{4}{|l|}{ Tumor pathology } \\
\hline Diameter of largest tumor (cm) & $3.9(2.4-6.6)$ & $3.0(2.2-4.3)$ & 0.062 \\
\hline Differentiation (Edmonson-Steiner grades I/II/III) & $0(0 \%) / 20(89 \%) / 14(41 \%)$ & $26(9 \%) / 226(74 \%) / 53(17 \%)$ & $0.002^{\mathrm{b}}$ \\
\hline Capsule formation (absent/partial/complete) & $6(18 \%) / 21(62 \%) / 7(21 \%)$ & $53(17 \%) / 161(53 \%) / 91(30 \%)$ & 0.505 \\
\hline Microvascular invasion & $28(82 \%)$ & $166(54 \%)$ & $0.002^{\mathrm{b}}$ \\
\hline Tumor multiplicity & $4(12 \%)$ & $33(11 \%)$ & 0.776 \\
\hline TNM stage (stages I/II/III) & $5(15 \%) / 27(79 \%) / 2(6 \%)$ & $117(38 \%) / 173(57 \%) / 15(5 \%)$ & $0.024^{\mathrm{b}}$ \\
\hline \multicolumn{4}{|l|}{ Immunoprofile, pathological finding in tissue microarray } \\
\hline K19 protein expression & $13(38 \%)$ & $22(7 \%)$ & $<0.001^{\mathrm{b}}$ \\
\hline EpCAM protein expression & $20(59 \%)$ & $97(32 \%)$ & $0.004^{\mathrm{b}}$ \\
\hline Tumor necrosis ( $>5 \%$ of the tumor core) & $29(85 \%)$ & $31(10 \%)$ & $<0.001^{\mathrm{b}}$ \\
\hline
\end{tabular}

Variables are expressed as median (interquartile range) or number (\%).

${ }^{\text {a }}$ Serum alpha-fetoprotein level not available for one patient.

b Indicates $P<0.05$.

${ }^{\mathrm{C}}$ Serum PIVKA-II level not available for 11 patients.

expression were significantly correlated with extrahepatic recurrence $(P<0.05$ for all, Supplementary Table 4 and Supplementary Figure 3E). As K19 and CAIX were shown to be significantly correlated with each other and CAIX had higher integrative area under the curve value than K19 (Supplementary Figure $3 \mathrm{~F}$ ), only CAIX was included in the subsequent multivariate analysis. On multivariate analysis, TNM stage and CAIX protein expression remained independent prognostic factors for extrahepatic recurrence $(P<0.05$ for all). To evaluate the added value of CAIX over the TNM stage, we performed integrative area under the curve analysis. The integrative area under the curve value of the TNM stage alone was 0.702 , and when combined with CAIX, the integrative area under the curve value increased to 0.736 . This increase in integrative area under the curve value was statistically significant (estimated difference: 0.035, 95\% confidence interval: 0.003-0.068; Figure 3d). Among hepatocellular carcinoma patients of the same TNM stage, hepatocellular carcinoma patients with CAIX expression showed shorter extrahepatic recurrence-free survival compared with those without (Figure 3e).

\section{Discussion}

Cancer stem cells have been demonstrated to have survival advantage to hypoxia in in vitro studies. ${ }^{4-7}$ Accordingly, a hypoxic tumor microenvironment may affect the generation and maintenance of cancer stem cells, which are implicated in the aggressive biologic behavior of tumors. ${ }^{17}$ Transarterial chemoembolization, one of the most popular locoregional therapy for hepatocellular carcinoma, specifically destroys tumor blood supply and induces severe hypoxia in tumors. ${ }^{13}$ The response to transarterial chemoembolization is predictive of recurrence and survival in post-transplantation patients ${ }^{18,19}$ and survival in non-surgical candidates. ${ }^{20,21}$ Despite the clinical significance of transarterial chemoembolization non-responders, little is known about the biologic background of these tumors.

To investigate the response to transarterial chemoembolization in hepatocellular carcinomas with stemness-related marker expression under hypoxic microenvironment, the expression status of CAIX, K19, or EpCAM and transarterial chemoembolization response (proportion of residual tumor in resected specimen) was evaluated in biopsytransarterial chemoembolization-resection-matched hepatocellular carcinomas (cohort 1). CAIX-positive hepatocellular carcinomas were also positive for K19 and EpCAM, and the incidence of CAIX, K19, or EpCAM expression was largely unchanged when compared before and after transarterial chemoembolization. Interestingly, all hepatocellular carcinomas with positive expression of CAIX, K19, or EpCAM in biopsy revealed variable extents of residual tumor, whereas most $(75 \%)$ hepatocellular carcinomas that did not express any of the three markers showed total necrosis in the resected specimens after transarterial chemoembolization. Taken together, hepatocellular 
carcinoma with expression of hypoxia-related marker (CAIX) or stemness-related makers (K19 and EpCAM) in biopsied tissue showed poor response to transarterial chemoembolization.

To further assess the difference in response to transarterial chemoembolization in hepatocellular carcinomas according to the expression status of hypoxia- and stemness-related markers in a larger number of cases, we studied explanted hepatocellular carcinomas with/without preoperative transarterial chemoembolization (cohort $2, n=85$ ). The positive rate of CAIX, K19, and EpCAM, and coexpression of these markers in residual tumors showed a significant increase in parallel with the number of transarterial chemoembolization sessions in cohort 2. Considering that the totally necrotic post-transarterial chemoembolization hepatocellular carcinoma cases were not included in cohort 2, the frequent expression of hypoxia- and stemness-related markers in residual hepatocellular carcinomas, which survived after multiple sessions of transarterial chemoembolization, could be considered to be related with transarterial chemoembolization resistance.

CAIX is a hypoxia-inducible component of the tumoral pH-regulatory system, and the CAIX promoter region contains a hypoxia-responsive element that is located immediately upstream of its transcription start site, which binds HIF $1 \alpha$. Indeed, HIF $1 \alpha$ is an exclusive regulator of CAIX activity in hypoxic microenvironments. ${ }^{3}$ The detection of HIF $1 \alpha$ is often challenging because of its fairly short half-life (less than 5 min). ${ }^{22}$ On the other hand, CAIX protein is more stable, and thus the detection of CAIX expression is more feasible, even by immunohistochemistry on paraffin-embedded tissues. In addition to its role as a marker of hypoxia, CAIX has been recently reported to be involved in tumor cell survival, adhesion, and migration in hypoxic microenvironments. ${ }^{23}$ Recently, it has been demonstrated that CD44(+)/CD24(-/low) cells were more frequently localized in CAIX-expressing areas of breast cancer, suggesting an association between cancer stem cells and hypoxia, ${ }^{24}$ and the depletion of CAIX has been reported to inhibit both the hypoxia-induced expansion of cancer stem cells in breast cancers. ${ }^{4}$ These findings suggest that CAIX may be a key factor in the regulation of stemness in hypoxic microenvironments. Accordingly, this study showed that stemness-related markers (K19 and EpCAM) and hypoxia marker (CAIX) expression levels were well correlated, and revealed that hepatocellular carcinomas with expression of K19, EpCAM, or CAIX showed significantly higher treatment resistance to transarterial chemoembolization with poor outcome. It is also worth noting that CAIX expression in hepatocellular carcinoma is often focal and patchy, and is preferentially expressed around the necrotic area. Therefore, assessment of CAIX expression in a limited amount of tissue, such as biopsy or tissue microarray, could result in underestimation of CAIX expression. In this study, two 2-mm-core biopsies were taken from different regions of individual hepatocellular carcinoma paraffin blocks for tissue microarray construction. The incidence of CAIX expression was $10 \%$ in cohort 3 (resected hepatocellular carcinomas without transarterial chemoembolization, tissue microarray) and $6 \%$ in cohort 2 (explanted hepatocellular carcinoma without transarterial chemoembolization, whole-tissue sections) and there was no significant difference between the two groups.

Currently, the Milan criteria, based on the size and number of tumors, are the benchmark selection criteria for determining an individual's suitability for liver transplantation. ${ }^{13}$ However, there is an increasing evidence that some patients who do not meet the criteria still show good survival rates after liver transplantation. ${ }^{25}$ Furthermore, among transplant recipients who do satisfy the Milan criteria, recurrences have been reported in $8-40 \%$, which in turn is associated with shorter overall survival. ${ }^{26,27}$ Therefore, the discovery of other factors that better reflect tumor biology, beyond the size and number of hepatocellular carcinomas, could improve the selection criteria for transplantation. A previous study reported that the responses to locoregional therapy was predictive for recurrence after transplantation. ${ }^{18}$ Recently, the progenitor cell signature (mainly K19 signature) was reported to be associated with poor outcome of hepatocellular carcinoma beyond the Milan criteria undergoing liver transplantation. ${ }^{28} \mathrm{In}$ our study, K19 and CAIX were expressed in $26 \%$ and $22 \%$, respectively, in residual hepatocellular carcinomas after transarterial chemoembolization compared with $6 \%$ and $6 \%$, respectively, in those without transarterial chemoembolization. The expression status of K19 and CAIX was well correlated, and the frequency of coexpression of these markers in residual hepatocellular carcinoma increased with a greater number of transarterial chemoembolization sessions. In fact, CAIX expression held additional value for predicting recurrence and survival among transplant recipients over the Milan criteria. Therefore, considering the very high cost of liver transplantation and shortage of donor livers, evaluation of markers for both stemness (K19, EpCAM) and hypoxic tumor microenvironment (CAIX) on biopsied tissue could potentially serve as a selection tool and prognostic marker for transplantation candidates, especially for those downstaged by transarterial chemoembolization.

Solid tumors including hepatocellular carcinoma often show spontaneous hypoxic necrosis, as the rapid growth of the tumors outstrips their blood supply. Therefore, we also evaluated hepatocellular carcinomas without any preoperative treatment (cohort $3, n=339$ ). In this cohort, CAIX expression was found in $10 \%$ of hepatocellular carcinomas and showed significant correlation with tumor necrosis, suggesting that CAIX is a good marker of a hypoxic tumor microenvironment. CAIX-positive hepatocellular carcinomas exhibited higher K19 and EpCAM expression, and more invasive pathological features. CAIX expression and TNM stage were independent 
predictors of extrahepatic recurrence, and the addition of CAIX to TNM stage significantly increased integrative area under the curve values. Therefore, it is considered that CAIX also has an additional value to predict the outcome for hepatocellular carcinomas without transarterial chemoembolization.

It is worth noting that the majority of our hepatocellular carcinoma cases were hepatitis B virus-related. Hepatitis B virus is frequently integrated into host genomes, and integration of $\mathrm{HBx}$ has been reported to be associated with the malignant transformation of hepatocytes, by affecting the transcriptional machinery. ${ }^{29}$ Recently, HBx was reported to enhance hypoxia signaling through HIF1 $\alpha$ activation, ${ }^{30}$ and EpCAM expression via activating $\beta$-catenin and regulating EpCAM promoter methylation. ${ }^{31,32}$ Thus, HBx might be an additional factor in the promotion of a switch in gene expression to stemness in hepatocarcinogenesis, especially under a hypoxic tumor microenvironment. Therefore, controlling stemness in hypoxic microenvironments may be more important in the treatment of hepatitis B virus-related hepatocellular carcinomas.

In conclusion, the expression levels of stemnessrelated (K19 and EpCAM) and hypoxia (CAIX) markers were correlated to each other, and hepatocellular carcinoma expressing these markers more frequently showed treatment resistance to transarterial chemoembolization, and poorer outcome compared with those without. This study also demonstrated that CAIX expression holds additional value in predicting patient outcomes after liver transplantation/resection over putative prognostic factors, including Milan criteria and TNM stage. Therefore, checking the expression status of markers both stemness (K19, EpCAM) and hypoxia (CAIX) may considered to have an additional value in predicting hepatocellular carcinoma outcome, especially for transarterial chemoembolization-treated cases.

\section{Acknowledgments}

We thank Keun Bae Bang for his help with immunohistochemistry and Anthony Thomas Milliken, ELS, for editing the manuscript. This research was supported by grants from the National Research Foundation of Korea (NRF) funded by the Korean government (MSIP) (grant number: NRF-2013R1A2A2A05005990, NRF-2012M3A9B6055350, NRF-2011-0030086).

\section{Disclosure/conflict of interest}

The authors declare no conflict of interest.

\section{References}

1 Vaupel P, Mayer A. Hypoxia in cancer: significance and impact on clinical outcome. Cancer Metastasis Rev 2007;26:225-239.
2 Semenza GL. Targeting HIF-1 for cancer therapy. Nat Rev Cancer 2003;3:721-732.

3 McDonald PC, Winum JY, Supuran CT et al. Recent developments in targeting carbonic anhydrase IX for cancer therapeutics. Oncotarget 2012;3:84-97.

4 Lock FE, McDonald PC, Lou Y et al. Targeting carbonic anhydrase IX depletes breast cancer stem cells within the hypoxic niche. Oncogene 2013;32:5210-5219.

5 Heddleston JM, Li Z, McLendon RE et al. The hypoxic microenvironment maintains glioblastoma stem cells and promotes reprogramming towards a cancer stem cell phenotype. Cell Cycle 2009;8:3274-3284.

6 Wang Y, Liu Y, Malek SN et al. Targeting HIF1alpha eliminates cancer stem cells in hematological malignancies. Cell Stem Cell 2011;8:399-411.

7 Muramatsu S, Tanaka S, Mogushi $\mathrm{K}$ et al. Visualization of stem cell features in human hepatocellular carcinoma reveals in vivo significance of tumor-host interaction and clinical course. Hepatology 2013;58:218-228.

8 Nguyen LV, Vanner R, Dirks P et al. Cancer stem cells: an evolving concept. Nat Rev Cancer 2012;12:133-143.

9 Kim H, Choi GH, Na DC et al. Human hepatocellular carcinomas with "Stemness"-related marker expression: keratin 19 expression and a poor prognosis. Hepatology 2011;54:1707-1717.

10 Seok JY, Na DC, Woo HG et al. A fibrous stromal component in hepatocellular carcinoma reveals a cholangiocarcinoma-like gene expression trait and epithelial-mesenchymal transition. Hepatology 2012; 55:1776-1786.

11 Bruix J, Sherman M. Management of hepatocellular carcinoma: an update. Hepatology 2011;53:1020-1022.

12 European Association For The Study Of The Liver, European Organisation For Research And Treatment Of Cancer. EASL-EORTC clinical practice guidelines: management of hepatocellular carcinoma. J Hepatol 2012;56:908-943.

13 Clavien PA, Lesurtel M, Bossuyt PM et al. Recommendations for liver transplantation for hepatocellular carcinoma: an international consensus conference report. Lancet Oncol 2012;13:e11-e22.

14 Chua TC, Liauw W, Saxena A et al. Systematic review of neoadjuvant transarterial chemoembolization for resectable hepatocellular carcinoma. Liver Int 2010;30: 166-174.

15 Midorikawa Y, Takayama T, Shimada K et al. Marginal survival benefit in the treatment of early hepatocellular carcinoma. J Hepatol 2013;58:306-311.

16 Takayama T, Makuuchi M, Kojiro $\mathrm{M}$ et al. Early hepatocellular carcinoma: pathology, imaging, and therapy. Ann Surg Oncol 2008;15:972-978.

17 Keith B, Simon MC. Hypoxia-inducible factors, stem cells, and cancer. Cell 2007;129:465-472.

18 Otto G, Schuchmann M, Hoppe-Lotichius M et al. How to decide about liver transplantation in patients with hepatocellular carcinoma: size and number of lesions or response to TACE? J Hepatol 2013;59: 279-284.

19 De Carlis L, Di Sandro S, Giacomoni A et al. Beyond the Milan criteria: what risks for patients with hepatocellular carcinoma progression before liver transplantation? J Clin Gastroenterol 2012;46:78-86.

20 Gillmore R, Stuart S, Kirkwood A et al. EASL and mRECIST responses are independent prognostic factors for survival in hepatocellular cancer patients treated with transarterial embolization. J Hepatol 2011;55: 1309-1316. 
21 Kim BK, Kim SU, Kim KA et al. Complete response at first chemoembolization is still the most robust predictor for favorable outcome in hepatocellular carcinoma. J Hepatol 2015;62:1304-1310.

22 Vordermark D, Kaffer A, Riedl S et al. Characterization of carbonic anhydrase IX (CA IX) as an endogenous marker of chronic hypoxia in live human tumor cells. Int J Radiat Oncol Biol Phys 2005;61:1197-1207.

23 McDonald PC, Dedhar S. Carbonic Anhydrase IX (CAIX) as a mediator of hypoxia-induced stress response in cancer cells. Subcell Biochem 2014;75:255-269.

24 Currie MJ, Beardsley BE, Harris GC et al. Immunohistochemical analysis of cancer stem cell markers in invasive breast carcinoma and associated ductal carcinoma in situ: relationships with markers of tumor hypoxia and microvascularity. Hum Pathol 2013;44: 402-411.

25 Yao FY, Xiao L, Bass NM et al. Liver transplantation for hepatocellular carcinoma: validation of the UCSFexpanded criteria based on preoperative imaging. Am J Transplant 2007;7:2587-2596.

26 Roayaie S, Schwartz JD, Sung MW et al. Recurrence of hepatocellular carcinoma after liver transplant: patterns and prognosis. Liver Transpl 2004;10:534-540.
27 Zimmerman MA, Ghobrial RM, Tong MJ et al. Recurrence of hepatocellular carcinoma following liver transplantation: a review of preoperative and postoperative prognostic indicators. Arch Surg 2008;143: 182-188, discussion 188.

28 Miltiadous O, Sia D, Hoshida Y et al. Progenitor cell markers predict outcome of patients with hepatocellular carcinoma beyond Milan criteria undergoing liver transplantation. J Hepatol 2015;63:1368-1377.

29 Guerrieri F, Belloni L, Pediconi N et al. Molecular mechanisms of HBV-associated hepatocarcinogenesis. Semin Liver Dis 2013;33:147-156.

30 Yoo YG, Na TY, Seo HW et al. Hepatitis B virus X protein induces the expression of MTA1 and HDAC1, which enhances hypoxia signaling in hepatocellular carcinoma cells. Oncogene 2008;27:3405-3413.

31 Arzumanyan A, Friedman T, Ng IO et al. Does the hepatitis $\mathrm{B}$ antigen $\mathrm{HBx}$ promote the appearance of liver cancer stem cells? Cancer Res 2011;71: 3701-3708.

32 Fan H, Zhang H, Pascuzzi PE et al. Hepatitis B virus X protein induces EpCAM expression via active DNA demethylation directed by RelA in complex with EZH2 and TET2. Oncogene 2016;35:715-726.

Supplementary Information accompanies the paper on Modern Pathology website (http://www.nature.com/ modpathol) 